\title{
Radiology Features of Chest Imaging and Its Correlation to Clinical Severity in Patients with COVID-19
}

\author{
Sarfraz Saleemi $^{1, ~ *}$, Fawaz Skaff ${ }^{2}$, Faisal Albaiz ${ }^{1}$, Reem Almaghrabi ${ }^{3}$, Fahad Alrabiah ${ }^{3}$, \\ Mohammed Alhajji ${ }^{1}$ \\ ${ }^{1}$ Department of Medicine, Section of Pulmonary Medicine, King Faisal Specialist Hospital \& Research Center, Riyadh, Saudi Arabia \\ ${ }^{2}$ Department of Radiology, Section of Cardiothoracic Imaging, King Faisal Specialist Hospital \& Research Center, Riyadh, Saudi Arabia \\ ${ }^{3}$ Department of Medicine, Section of Infectious Diseases, King Faisal Specialist Hospital \& Research Center, Riyadh, Saudi Arabia
}

\section{Email address:}

ssaleemi@kfshrc.edu.sa (S. Saleemi)

${ }^{*}$ Corresponding author

\section{To cite this article:}

Sarfraz Saleemi, Fawaz Skaff, Faisal Albaiz, Reem Almaghrabi, Fahad Alrabiah, Mohammed Alhajji. Radiology Features of Chest Imaging and Its Correlation to Clinical Severity in Patients with COVID-19. International Journal of Medical Imaging. Vol. 8, No. 2, 2020 , pp. 35-38. doi: $10.11648 /$ j.jimi.20200802.13

Received: May 27, 2020; Accepted: June 10, 2020; Published: June 20, 2020

\begin{abstract}
Background: COVID-19 caused by SARS CoV-2 involves respiratory system leading to respiratory failure and Acute Respiratory Distress Syndrome (ARDS) in critical patients. Several chest imaging features have been reported in patients with COVID-19 ranging from focal to diffuse lung opacities. There is no data from Saudi Arabia on the chest imaging findings in these patients. Material and Methods: CT chest data of 29 patients who were admitted with confirmed diagnosis of COVID-19 by real-time reverse transcriptase polymerase chain reaction (RT-PCR) was reviewed. Radiology abnormality was categorized based upon pattern and distribution. Clinical and laboratory data of patients were collected by reviewing the electronic medical record. Patients were divided into mild and severe group based on clinical assessment and laboratory criteria. Radiology changes were compared with disease severity. Results: Median (Q1, Q3) age was $58(41,70)$ years and median $(\mathrm{Q} 1, \mathrm{Q} 3)$ time from symptom onset to CT scan was 6.5 $(3.0,9.75)$ days. Bilateral ground glass opacities were the most common CT scan feature in patients with COVID-19 (76\%). Opacities were dominant in the lower zone (72\%) and frequently distributed peripherally (48\%). Severe disease was most likely to have bilateral opacities compared with mild $(p=0.001)$ and it was correlated with rise of inflammatory markers, Ferritin $(\mathrm{p}=0.014)$ and $\mathrm{C}$-reactive protein $(\mathrm{p}=0.0003)$. Conclusion: Most patients with COVID-19 who have abnormal CT scan of chest show ground glass opacities. Bilateral opacities are more common in severe disease and is correlated with elevated inflammatory markers.
\end{abstract}

Keywords: COVID-19, SARS CoV-2, CT Scan, Ground Glass, Opacity

\section{Introduction}

COVID-19 caused by SARS-CoV2 was declared a global pandemic on March 11, 2020 by World Health Organization. [1, 2] Mortality in patients who develop Acute Respiratory Distress Symptoms is high. The most common symptoms reported in COVID-19 patients are fever, cough and dyspnea. [3,4] Severity of disease is classified based on clinical, laboratory and radiology characteristics. [5] There is a wide spectrum of chest imaging abnormalities described in COVID-19 patients ranging from ground glass, focal consolidation, nodular and interstitial opacities. The different pattern of chest imaging may show during the course of illness. The extent of radiology involvement plays a vital role in disease assessment, prognosis and follow up of patients. Involvement of $>50 \%$ of lung fields on CT scan is shown to be associated with poor prognosis. The correlation between CT findings and disease severity and mortality can be concluded from a number of studies. [6,7]

This is the first study from Saudi Arabia describing radiology patterns and its association with disease severity in COVID-19 patients. 


\section{Methods}

\subsection{Study Design and Patient Characteristics}

This was a retrospective study at a tertiary care hospital. Adult patient of $>18$ year of age who were admitted with a laboratory confirmed diagnosis of COVID-19 were included in the study. The diagnosis was confirmed by real time polymerase chain reaction (RT-PCR) (Altona, HamburgGermany) by using nasopharyngeal swab. Clinical data of consecutive 50 patients admitted from March 13, 2020 onward were reviewed. Twenty-nine patients who had CT scan of chest were included in the study. The study was approved by research and ethics regulatory committee of the institution. Informed consent was waived in view of retrospective observational nature of study.

\subsection{Data Collection}

Data was collected retrospectively from electronic record of patients. Confidentiality of data was ensured by hiding the medical record number of patients from data handlers. Information was collected on demographic, symptomatology, disease severity, laboratory measurements, radiology imaging, treatment and clinical outcome. The disease severity was classified into mild and severe based on clinical and laboratory parameters. Patients with mild symptoms, modest rise in inflammatory markers such as C-reactive protein and ferritin and normal oxygen saturation were classified as mild while severe disease was defined as respiratory symptoms, marked rise in inflammatory markers and low oxygen saturation compared to baseline. Radiology imaging of both groups were analyzed.

\subsection{Radiology Data}

All study patients had Chest X-ray on admission and CT scan was done in patients who had suspicion of abnormality on chest X-ray or patient with high risk clinical and laboratory characteristics. Non-contrast CT scan of chest was done in supine position in full inspiration and images were acquired in lung and mediastinal window. The images were reviewed and reported by experienced thoracic radiologist on Sectra's PACS imaging system (Sectra Group). CT images were assessed for specific radiology patterns including ground glass (hazy opacity not obscuring the underlying vasculature), consolidation, mixed ground glass and consolidation, reticular pattern, atelectatic bands, nodular opacities and bronchial wall thickening. Distributions of opacities were divided into focal, bilateral, diffuse and peripheral. Dominant zone occupying the opacity were assessed by dividing each lung into three zones, upper, middle and lower. Upper zone being above the carina, lower below the inferior pulmonary vein and middle in-between.

\subsection{Statistical Analysis}

Data was summarized by using descriptive statistics. Results were reported as median with inter-quartile range and mean with standard deviation and categorical variable were calculated as counts (n) and percentages (\%). Differences between groups were analyzed using Mann-Whitney U test, two tailed Z-test and T-test where appropriate. $\mathrm{P}$ value of $<0.05$ was considered to be statistically significant.

\section{Results}

\subsection{Clinical characteristics}

Twenty-nine patients who had CT scan were included in the study. The diagnosis of COVID-19 was confirmed by real time polymerase chain reaction (RT-PCR) for SARS-CoV2. Median (Q1, Q3) age of patients was $58(41,70)$ and $14(48 \%)$ were male. Eight (28\%) patients were smoker, either current or past. The most common symptoms were cough, fever and fatigue. Ten (34\%) patients were clinically categorized as mild while $19(66 \%)$ had severe disease. Clinical and laboratory characteristics of patients are summarized in Table 1.

Table 1. Clinical characteristics and laboratory data.

\begin{tabular}{ll}
\hline & $\mathbf{n}=\mathbf{2 9}$ \\
\hline Demographics & \\
Age, years, median $(\mathrm{Q} 1, \mathrm{Q} 3)$ & $58(41,70)$ \\
Female, $\mathrm{n}(\%)$ & $15(52)$ \\
Male, $\mathrm{n}(\%)$ & $14(48)$ \\
Smoker or ex-smoker, n (\%) & $8(28)$ \\
Comorbid conditions, overall, n (\%) & $22(76)$ \\
$>2$ comorbid conditions, $\mathrm{n}(\%)$ & $16(73)$ \\
Clinical symptoms, $\mathrm{n}(\%)$ & \\
Fever & $21(72)$ \\
Cough & $17(59)$ \\
Dyspnea & $12(31)$ \\
Laboratory values, unit, normal value, & \\
median $(\mathrm{Q} 1, \mathrm{Q} 3)$ & \\
White blood cell, $\times 10^{9} / \mathrm{L},(3.9-11.0)$ & $5.1(3.37,6.12)$ \\
Neutrophil, $\times 10^{9} / \mathrm{L},(1.35-7.50)$ & $3.12(1.91,4.17)$ \\
Lymphocyte, $\times 10^{9} / \mathrm{L},(1.50-4.30)$ & $0.33(0.97,1.57)$ \\
Neutrophil/Lymphocyte ratio, $(1.7 \pm 0.7)$ & $2.64(1.22,3.96)$ \\
Platelet count, $\times 10^{9} / \mathrm{L},(155-435)$ & $184(148,249)$ \\
Ferritin, $\mu \mathrm{g} / \mathrm{L},(13.0-150)$ & $243(58,442)$ \\
C-reactive protein, mg/L, $(<3.0)$ & $13.75(3.12,38.75)$ \\
D-dimer, $\mu \mathrm{g} / \mathrm{mL},(0.0-0.50)$ & $0.51(0.31,1.01)$ \\
Disease severity, $\mathrm{n}(\%)$ & $10(34)$ \\
Mild & $19(66)$ \\
Severe & $6.5(3.0,9.75)$ \\
Symptom onset to CT scan, days, median $(\mathrm{Q} 1, \mathrm{Q} 3)$ & \\
\hline
\end{tabular}

\subsection{Chest Imaging}

Median time (Q1, Q3) from symptom onset to CT scan was $6.5(3.0,9.75)$ days. Three patients $(10 \%)$ had normal CT scan. Focal ground glass (GG) opacity was present in $5(17 \%)$ patients, mixed GG and consolidation (figure 1) in 13 (45\%), bilateral GG (figure 2) in $21 \quad(72 \%)$, and nodular/reticular/band like opacities (figure 3) in 8 (28\%) patients. The opacities had predominantly peripheral (figure 4) distribution in $14(48 \%)$ and lower zones in $21(72 \%)$ (Table 2). Bilateral opacities were more common in clinically severe disease 18 (95\%) compared with mild 3 (30\%). Inflammatory markers, $\mathrm{C}$-reactive protein (CRP) and ferritin were significantly elevated in severe disease $p=0.0003$ and 
$p=0.014$ respectively. (Table 3 )

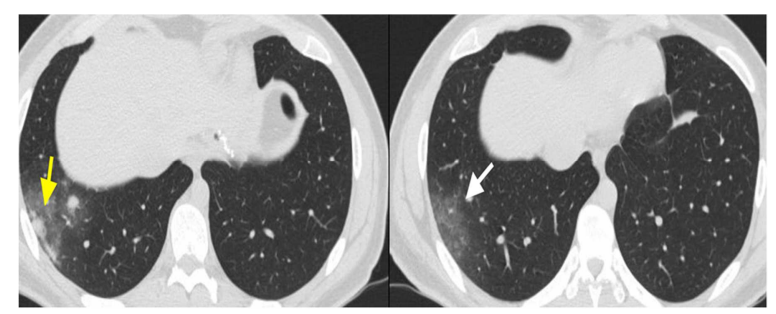

Figure 1. CT scan of a 21-year old male with no co-morbid medical conditions presented with fever, cough and sore throat. Focal ground glass (White arrow) and mixed focal ground glass and consolidation (yellow arrow).

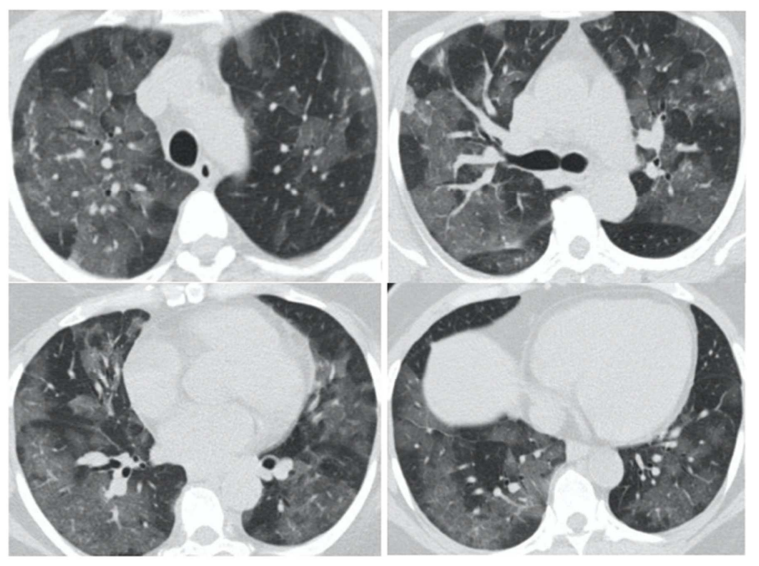

Figure 2. CT Scan of a 48-year old female with history of hypertension and laboratory confirmed COVID-19 presented with dry cough and dyspnea for one week. Axial images show diffuse bilateral ground glass opacities.

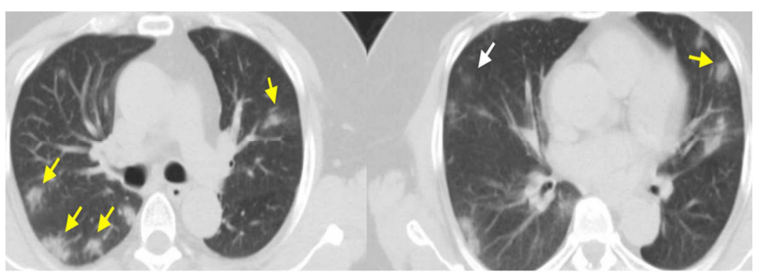

Figure 3. CT scan of a 58-year old male with history of hypertension presented with 4-day history of dry cough and fever. Axial images show bilateral nodular consolidation (yellow arrows) and focal ground glass opacities (white arrow).

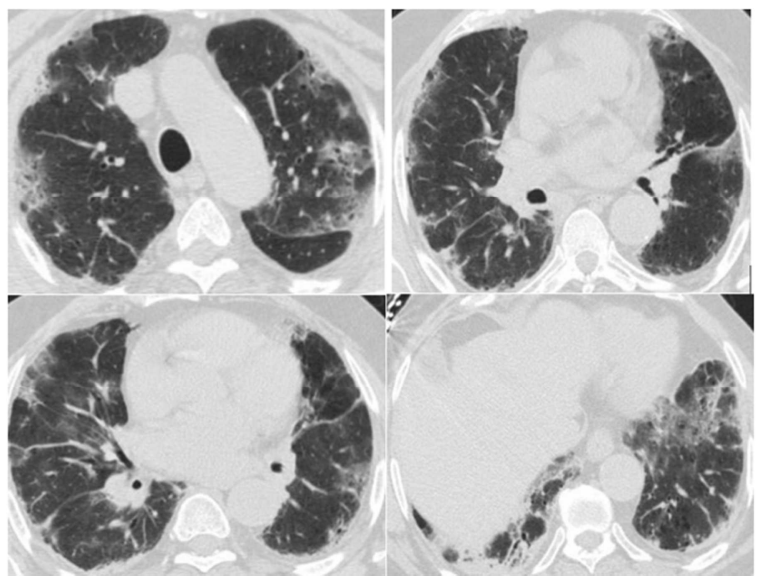

Figure 4. Peripheral distribution of ground glass opacities and interstitial thickening.
Table 2. CT imaging features in COVID-19 patients.

\begin{tabular}{lllll}
\hline & $\begin{array}{l}\text { Overall } \\
\mathbf{n = 2 9}\end{array}$ & $\begin{array}{l}\text { Mild } \\
\mathbf{n = 1 0}\end{array}$ & $\begin{array}{l}\text { Severe } \\
\mathbf{n = 1 9}\end{array}$ & P value \\
\hline CT scan abnormality, n (\%) & & & & \\
Clear & $3(10)$ & $3(30)$ & $0(0)$ & .0117 \\
Focal GG* & $5(17)$ & $4(40)$ & $1(5)$ & .0187 \\
Bilateral GG & $21(72)$ & $3(30)$ & $18(95)$ & .0002 \\
Mixed Consolidation \& GG & $13(45)$ & $4(40)$ & $9(47)$ & .7039 \\
Reticular/nodule/band & $8(28)$ & $2(20)$ & $6(32)$ & .5092 \\
Dominant CT distribution, n (\%) & & & & \\
Upper/Middle zone & $4(14)$ & $1(10)$ & $3(16)$ & .6672 \\
Lower zone & $21(72)$ & $6(60)$ & $15(79)$ & .2757 \\
Peripheral & $14(48)$ & $2(20)$ & $12(63)$ & .0271 \\
Non-selective & $7(24)$ & $1(10)$ & $6(32)$ & .1970 \\
\hline
\end{tabular}

*GGO-ground glass opacity

Table 3. Correlation of imaging features to clinical severity and inflammatory markers.

\begin{tabular}{llll}
\hline & \multicolumn{3}{l}{ Disease severity } \\
\cline { 2 - 4 } & \multicolumn{1}{l}{ Mild $\boldsymbol{n}=\mathbf{1 0}$} & Severe $\boldsymbol{n}=\mathbf{1 9}$ & P value \\
\hline CT abnormality, n (\%) & $3(30)$ & $0(0)$ & .0117 \\
Clear & $4(40)$ & $1(5)$ & .0187 \\
Focal & $3(30)$ & $18(95)$ & .0002 \\
Bilateral & & & \\
Inflammatory markers, unit, & & 296 & \\
normal value, median (Q1, Q3) & & $(184,672)$ & .0142 \\
Ferritin, $\mu \mathrm{g} / \mathrm{L},(13.0-150)$ & $58(44,233)$ & & \\
C-reactive protein, $\mathrm{mg} / \mathrm{L},(<3.0)$ & $2.7(1.45,5.8)$ & $31(12.7,70)$ & .0003 \\
\hline
\end{tabular}

\section{Discussion}

Chest imaging has an important role in diagnosis and follow up of COVD-19 patients. Several radiology patterns have been reported. CT scan in COVID-19 patients show diverse changes including ground glass, consolidation, nodules and reticular changes. Some studies have reported temporal CT changes on follow up examination such as transition from ground glass to superimposition of consolidation. Chest imaging pattern in this study matches the radiology features described in recent COVID-19 literature. $[8,9,10]$ Only $3(10 \%)$ patients in this study had normal CT scan although Guan [11] has reported 23.6\% laboratory confirmed COVID-19 patients with normal CT scan. The dominant radiology feature in this cohort was ground glass opacity which, in most patients, was bilateral with peripheral distribution. Similar finding has been reported by Shuchang Zhou [12] where 48 (77.4\%) of 62 patients had peripheral distribution of opacities. The pathophysiology behind peripheral distribution of opacities is not very well understood. However, there is close relation between the pattern of CT scan findings and disease severity. The correlation between CT findings and disease severity and mortality can be concluded from a number of studies. Involvement of $>50 \%$ of lung fields on CT scan is shown to be associated with poor prognosis. [13, 14, 15] In this study, the pattern of bilateral opacities showed correlation with 
disease severity and elevated levels of inflammatory markers.

\section{Conflict of Interest}

\section{None}

\section{Consent}

Informed consent was waived by the institutional Review Board due to the retrospective nature of the study and urgent need to collect data.

\section{Ethical Approval}

Approval was granted by Ethics Committee of King Faisal Specialist Hospital \& Research Center.

\section{References}

[1] WHO. Coronavirus disease (COVID-19) outbreak. 2020. https://www.who.int/emergencies/diseases/novel-coronavirus2019 (accessed Apr 30, 2020).

[2] N. Zhu, D. Zhang, W. Wang, et al. A novel coronavirus from patients with pneumonia in China, 2019. N Engl J Med, 382 (8) (2020), pp. 727-733.

[3] Huang C, Wang Y, Li X, Ren L, Zhao J, Hu Y, et al. Clinical features of patients infected with 2019 novel coronavirus in Wuhan, China. Lancet. 2020 Feb 15; 395 (10223): 497-506.

[4] Chen N, Zhou M, Dong X, Qu J, Gong F, Han Y, et al. Epidemiological and clinical characteristics of 99 cases of 2019 novel coronavirus pneumonia in Wuhan, China: a descriptive study. Lancet. 2020 Feb 15; 395 (10223): 507-513.

[5] Wang D, Hu B, Hu C, Zhu F, Liu X, Zhang J, et al. Clinical Characteristics of 138 Hospitalized Patients With 2019 Novel Coronavirus-Infected Pneumonia in Wuhan, China. JAMA. 2020; 323 (11): 1061-1069.

[6] Pan F, Ye T, Sun P, Gui S, Liang B, Li L, et al. Time Course of Lung Changes On Chest CT During Recovery From 2019
Novel Coronavirus (COVID-19) Pneumonia. Radiology 2020 295: 3, 715-721.

[7] Wu J, Wu X, Zeng W, et al. Chest CT Findings in Patients With Coronavirus Disease 2019 and Its Relationship With Clinical Features. Invest Radiol. 2020; 55 (5): 257-261.

[8] Yueying Pan, Hanxiong Guan, Shuchang Zhou, Yujin Wang, Qian Li, Tingting Zhu, et al. Initial CT findings and temporal changes in patients with the novel coronavirus pneumonia (2019-nCoV): a study of 63 patients in Wuhan, China. European Radiology 2020, 30: 3306-3309.

[9] Wei Zhao, Zheng Zhong, Xingzhi Xie, Qizhi Yu, Jun Liu. Relation Between Chest CT Findings and Clinical Conditions of Coronavirus Disease (COVID-19) Pneumonia: A Multicenter Study. American Journal of Roentgenology, 2020; 214: 5, 1072-1077.

[10] Z. Cheng, Y. Lu, Q. Cao, et al. Clinical features and chest CT manifestations of coronavirus disease 2019 (COVID-19) in a single-center study in Shanghai, China. Am J Roentgenol (2020), pp. 1-6.

[11] Guan WJ, Ni ZY, Hu Y, et al. Clinical Characteristics of Coronavirus Disease 2019 in China. N Engl J Med. 2020; 382 (18): $1708-1720$

[12] Shuchang Zhou, Yujin Wang, Tingting Zhu, Liming Xia. CT Features of Coronavirus Disease 2019 (COVID-19) Pneumonia in 62 Patients in Wuhan, China. AJR 2020; 214: $1-8$.

[13] Meng H, Xiong R, He R, et al. CT imaging and clinical course of asymptomatic cases with COVID-19 pneumonia at admission in Wuhan, China. J Infect. 81 (2020); 33-39.

[14] Ran Yang, Xiang Li, Huan Liu, Yanling Zhen, Xianxiang Zhang, Qiuxia Xiong, Yong Luo, Cailiang Gao, and Wenbing Zeng. Chest CT Severity Score: An Imaging Tool for Assessing Severe COVID-19. Radiology: Cardiothoracic Imaging. 2020; 2: 2.

[15] Yin X, Min X, Nan Y, Feng Z, Li B, Cai W, Xi X, Wang L. Assessment of the Severity of Coronavirus Disease: Quantitative Computed Tomography Parameters versus Semiquantitative Visual Score. Korean J Radiol. 2020 Jan; 21: e91. 